\title{
LAS OSCILACIONES CLIMATICAS RECIENTES EN ZARAGOZA (1865-1984)
}

\author{
José M $M^{a}$ CUADRAT PRATS
}

Universidad de Zaragoza

\begin{abstract}
RESUMEN: Las temperaturas de Zaragoza, durante los ciento veinte años de observaciones han conocido un aumento significativo hasta 1950. En este momento la tendencia cambia y, mientras las máximas inician un descenso, las minimas mantienen un alza sostenida, en particular las de invierno. La explicación de este fenómeno se encuentra en la evolución climática seguida por el hemisferio Norte y en la posible influencia urbana.
\end{abstract}

\begin{abstract}
Zaragoza's temperatures -during the last 120 years of observations- have experienced a significant increase till 1950. Then the tendency changes and, while the maximum temperatures begin to fall, the minimun ones keep going up, particularly throung the winter. Explanation to this phenomenon lies in the climatic evolutian followed by the North hemisphere and in a possible urban influence.
\end{abstract}

Sumario: Introducción.- Características térmicas de Zaragoza.- Tendencia de las temperaturas anuales.- Tendencia estacional e influencia urbana.- Conclusión.- Bibliografía citada.

\section{INTRODUCCION}

Uno de los campos más actuales de la investigación climática y a la vez más inciertos por la dificultad de su estudio, es sin duda el relativo al conocimiento de la variabilidad del clima, de sus posibles causas y las especulaciones acerca de su evolución. Del interés que suscita son una buena prueba las numerosas referencias bibliográficas existentes (POULSIN, MOUNIER, GREGORY, son sólo algunas referencias obligadas), las reuniones científicas de carácter internacional consagradas a los cambios climáticos recientes (Sección 3 del XV Congreso Internacional de Geografía, celebrado en París en 1980, y su continuación en la Ponencia XVI del Congreso celebrado en Australia en 1984), o la puesta en marcha por la Organización Metcorológica Mundial, en colaboración con otros organismos internacionales, del Programa Mundial de Investigaciones Climáticas para estudiar el cambio climático y su impacto sobre las actividades humanas. También en España, el Instituto Nacional de Meteorología ha fijado como tema prioritario de estudio la variabilidad climática y, de igual modo, éste es el centro de interés de recientes investigaciones españolas, como lo prueban los trabajos de CREUS y PUIGDEFABREGAS (1981 y 1983), CLAVERO (1982), LOPEZ GOMEZ y FERNANDEZ (1986), MORENO y MARTIN VIDE (1986), RASO (1987), GONZALEZ HIDALGO (1989) y los trabajos del Simposio sobre fluctuaciones climáticas del Cuaternario, celebrado en 1986. En este línea de trabajo, presentamos un avance de resultados de la evolución térmica de Zaragoza, como aproximación a un estudio más amplio que pretende conocer las características y la variabilidad del clima de la ciudad y su entorno, y los posibles cambios debidos a la influencia del área urbana. 


\section{CARACTERISTICAS TERMICAS DE ZARAGOZA}

La falta de registros continuos y la brevedad de la información meteorológica ha sido siempre un grave problema en todo trabajo climático. Por fortuna, el observatorio de Zaragoza dispone de una larga serie de registros, fiables, homogéneos y con escasas lagunas, que permiten remontar el estudio hasta mediados del siglo pasado. Su antigüedad data de 1855 , con la puesta en funcionamiento de los primeros instrumentos colocados en un torreón del edificio actualmente derruido de la Universidad Literaria de Zaragoza; más tarde, en 1892 y 1942, sufre dos cambios de ubicación, no muy distantes y bien conocidos, manteniendo siempre análogas condiciones de instalación: en el centro del casco urbano y en lo alto de una torre. En 1984 es trasladado al interior del Parque Primo de Rivera, donde la presencia de extensa superficie arbolada y amplio espacio ajardinado, modifican sustancialmente las iniciables características de situación y recomiendan no hacer uso de estos datos. Igualmente se ha hecho con los registros de los primeros diez años por carecer de total fiabilidad.

Con los ciento veinte años considerados (1865-1984), se ha elaborado una somera caracterización térmica a partir del cálculo estadístico del valor medio, la desviación típica, el coeficiente de variación y el valor máximo y mínimo de las temperaturas medias anuales, las temperaturas medias de las máximas y las temperaturas medias de las mínimas, y que hemos recogido en el cuadro 1:

$$
T^{\circ} \text { media anual } \quad T^{\mathbb{0}} \text { media máximas } \quad T^{\mathbf{0}} \text { media mínimas }
$$

$\begin{array}{lccc}\text { Media } & 14^{\prime} 73 & 20^{\prime} 04 & 9^{\prime} 28 \\ \text { Desviación Típica } & 0^{\prime} 62 & 0^{\prime} 69 & 0^{\prime} 79 \\ \text { Coef. Variación (\%) } & 4^{\prime} 23 & 3^{\prime} 43 & 8^{\prime} 49 \\ \text { Valor máximo } & 16^{\prime} 1 & 22^{\prime} 0 & 0^{\prime} 8 \\ \text { Valor mínimo } & 13^{\prime} 4 & 18^{\prime} 3 & 6^{\prime} 7\end{array}$

Cuadro 1. Valores estadísticos básicos de las temperaturas de Zaragoza (1865-1984)

Estos valores promedios son muy significativos de la temperatura zaragozana y ayudan a definir su ambiente atmosférico, sin embargo, la realidad cambiante del clima debe buscarse en el ritmo de sus valores, que a lo largo del período analizado muestran una tendencia hacia la suavización, en particular de las temperaturas de invierno, acorde con la evolución apreciada en el hemisferio Norte desde 1800 (según LANDSBERG, 1984, p. 279), tal como se examina a continuación.

\section{TENDENCIAS DE LAS TEMPERATURAS ANUALES}

Los valores térmicos de Zaragoza, desde que existen registros continuos, presentan estas tendencias: ciclos alternantes creciente-decreciente de las temperaturas medias, con cierto descenso en los últimos años, variaciones similares en las temperaturas máximas y acusado aumento de las mínimas. Así se deduce del análisis de los datos disponibles. No obstante, 


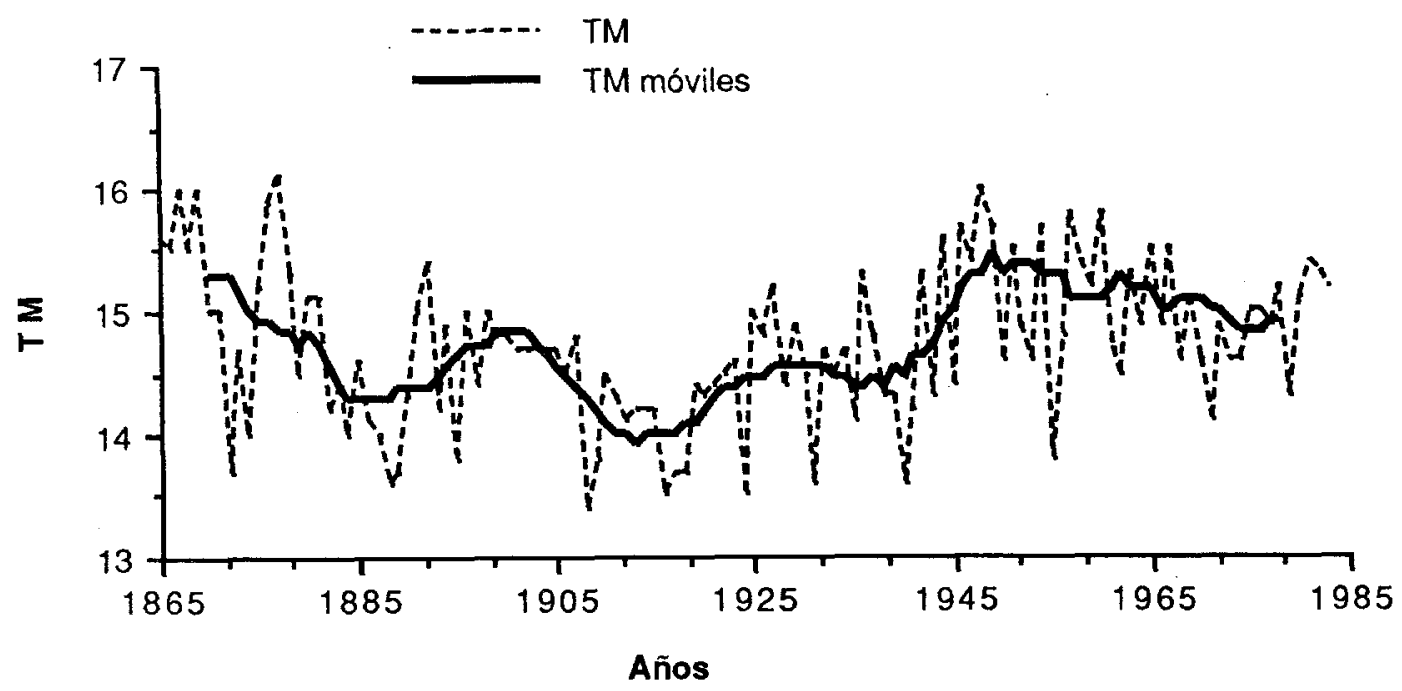

Fig. 1. Temperaturas medias anuales (TM) de Zaragoza (1865-1984) y sus médias móviles de 11 años.

estas cifras estadísticas, aún estando ordenadas cronológicamente, muestran pequeñas fluctuaciones o "dientes de sierra" que dificultan la puesta en evidencia de esta evolución; por ello se ha procedido a suavizar las irregularidades más esporádicas e irrelevantes de las series iniciales mediante el método de las medias móviles, centradas en este caso de 11 en 11 años, con las que se facilita la observación de posibles tendencias enmascaradas en el conjunto de la serie. Los resultados, expresados de forma gráfica, se presentan en las figuras 1 y 2 sobrepuestas a las temperaturas anuales.

Las medias móviles de las temperaturas medias sugieren una evolución cíclica que se inicia con un período decreciente hasta 1914, seguido por otro de ascenso que culmina en torno a 1950 y de nuevo una etapa de suave descenso hasta el momento actual. En efecto, desde el comienzo de los datos hasta el segundo decenio del presente siglo las temperaturas disminuyeron cerca de $1^{\prime} 5^{\circ}$ y se situaron en $13^{\prime} 9^{\circ}$ y $14^{\circ}$ en los años 1914 y 1915 respectivamente; cifras que no se han vuelto a repetir hasta el presente. A partir de aquí se mantiene una tónica alcista de $1^{\prime} 4^{\circ}$, hasta 1950 , y prácticamente se recuperan los valores alcanzados en el pasado siglo. En los años siguientes se ha mantenido un moderado descenso, entre $0^{\prime} 5^{\circ}$ y $0^{\prime} 6^{\circ}$, bien definido hasta la década de los setenta e interrumpido en parte en los últimos años, sin que podamos mostrar una tendencia climática significativa. De cada período se ha querido conocer su significación estadística y se ha procedido a determinar la recta de tendencia por cl método de los mínimos cuadrados, previo cálculo del coeficiente de correlación 

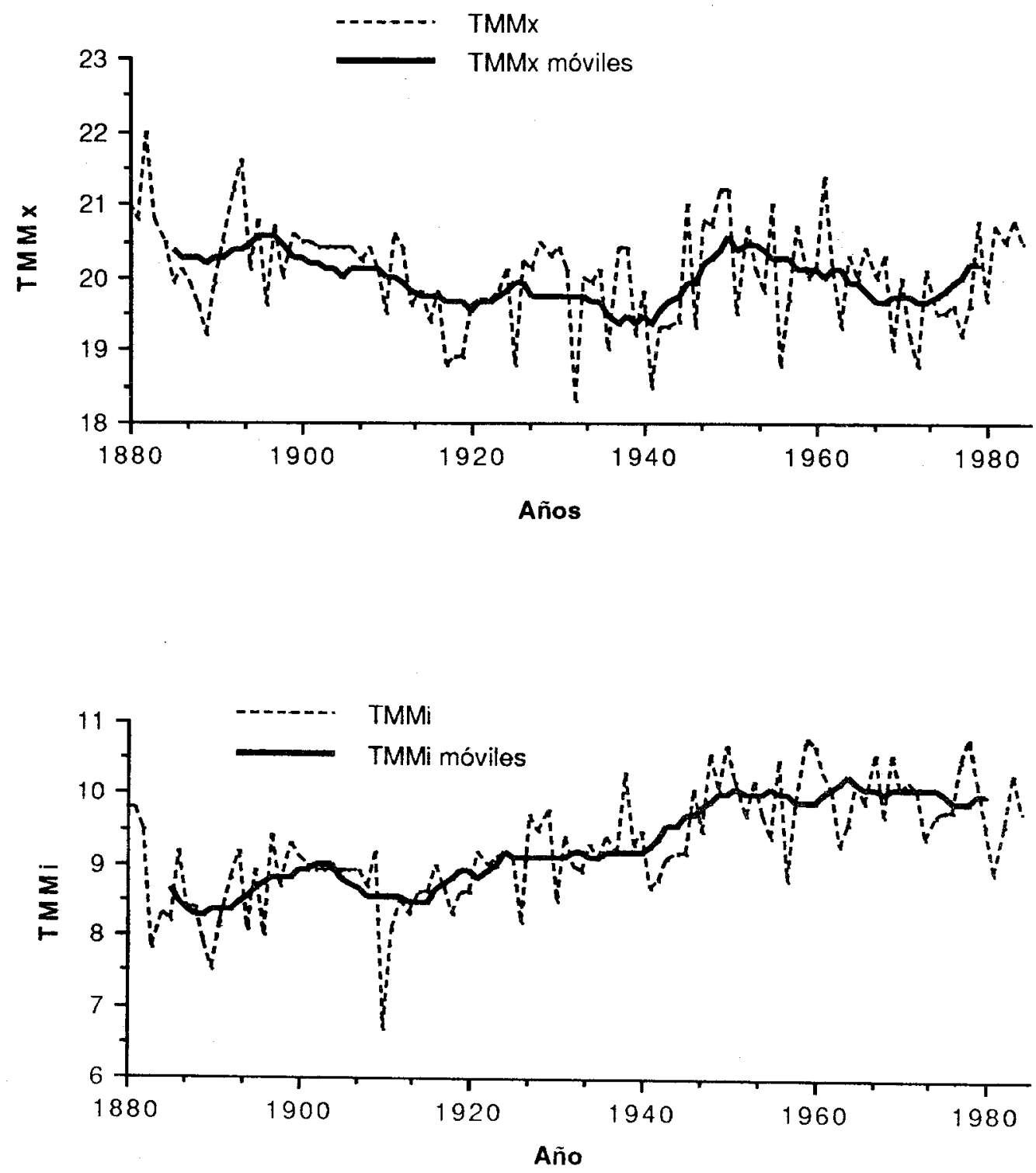

Fig. 2. Temperaturas medias de las máximas (TMMx) y temperaturas medias de las mínimas (TMMi) de Zaragoła (1880-1984) y sus medias móviles de 11 años 
entre los números ordinales de los años estudiados y las respectivas temperaturas móviles; los resultados son los siguientes:

\begin{tabular}{lcc} 
Período & Correlación $(\sigma)$ & \multicolumn{2}{c}{ Recta de tendencia } \\
$1870-1979$ & $0^{\prime} 35$ & $\mathfrak{t}_{\mathbf{i}}=14^{\prime} 47+0^{\prime} 003 \mathbf{x}_{\mathbf{i}}$ \\
$1870-1914$ & $-0^{\prime} 73$ & $\mathbf{t}_{\mathbf{i}}=14^{\prime} 99-0^{\prime} 017 \mathbf{x}_{\mathbf{i}}$ \\
$1915-1950$ & $0^{\prime} 9$ & $\mathbf{t}_{\mathbf{i}}=1^{\prime} 05+0^{\prime} 028 \mathbf{x}_{\mathbf{i}}$ \\
$1951-1979$ & $-0^{\prime} 81$ & $\mathbf{t}_{\mathbf{i}}=15^{\prime} 19-0^{\prime} 014 \mathbf{x}_{\mathbf{i}}$
\end{tabular}

en los que $\sigma$ es el coeficiente de correlación de Pearson, $t_{i}$ la temperatura media anual teórica del año $i, y$ y $x_{i}$ equivale sucesivamente a $1,2,3 \ldots$ para los años $1870,1871,1872, \ldots$

Las temperaturas medias de las máximas trazan a grandes rasgos el mismo perfil: curva decreciente hasta 1920 , con descenso de $1^{\prime} 1^{\circ}$, recuperación paulatina hasta 1950 , de poco más de $1^{\circ}$, y nueva disminución hasta 1973, donde se invierten los valores. De este modo se dibujan también tres grandes ciclos, sin diferencia apreciable en su tendencia con los ritmos seguidos por las temperaturas medias. El comportamiento más simple, y a la vez el más llamativo, es el seguido por las temperaturas medias de las mínimas, que no han dejado de incrementarse desde el pasado siglo, variando desde los $8^{\prime} 29^{\circ}$ del año 1887 , a los $10^{\prime} 11^{\circ}$ alcanzados en 1973, y estabilizándose a partir de este momento; es decir, un considerable aumento, de $1^{\prime} 8^{\circ}$, en un período de tiempo relativamente corto, que se asemeja a los $1^{\prime} 76^{\circ}$ hallados en Madrid por LOPEZ GOMEZ y FERNANDEZ (1986), en un número de años muy similar y a partir de medias móviles quinquenales.

En conjunto, los cambios experimentados por las temperaturas de Zaragoza, son muy similares a los observados en otras ciudades españolas como Madrid, Barcelona o Roquetas, y al de otras localidades relativamente más alejadas como las francesas (ESCOURROU, G. y P., 1984). También en ellas las notas características son las alternancias frías y cálidas en las temperaturas máximas, con cierta tendencia a disminuir pero no de forma clara en los años más recientes, y, sobre todo, el continuado aumento de las mínimas. Así, en Nantes, en lo que va de siglo, la temperatura mínima se ha incrementado en $12^{\circ}$, y valores similares se hallan en Caen o Marsella. Las máximas anuales, por el contrario, en estos observatorios tienden a disminuir, pero siempre con cifras muy bajas o con tendencias actuales difíciles de seguir, como son los casos de Madrid, Barcelona o Zaragoza.

\section{TENDENCIA ESTACIONAL E INFLUENCIA URBANA}

La evolución térmica analizada coincide en buena medida con la dinámica del clima del hemisferio Norte, donde los largos registros señalan un aumento térmico hasta 1930-1950 y un enfriamiento posterior. Así se comprueba en los valores anuales. Pero, sin duda, las temperaturas más afectadas por este cambio han sido las estivales y las invernales, particularmente en el curso de este siglo donde parecen llevar caminos contrarios: suavización de las máximas de verano y caldeamiento de las mínimas de invierno. 

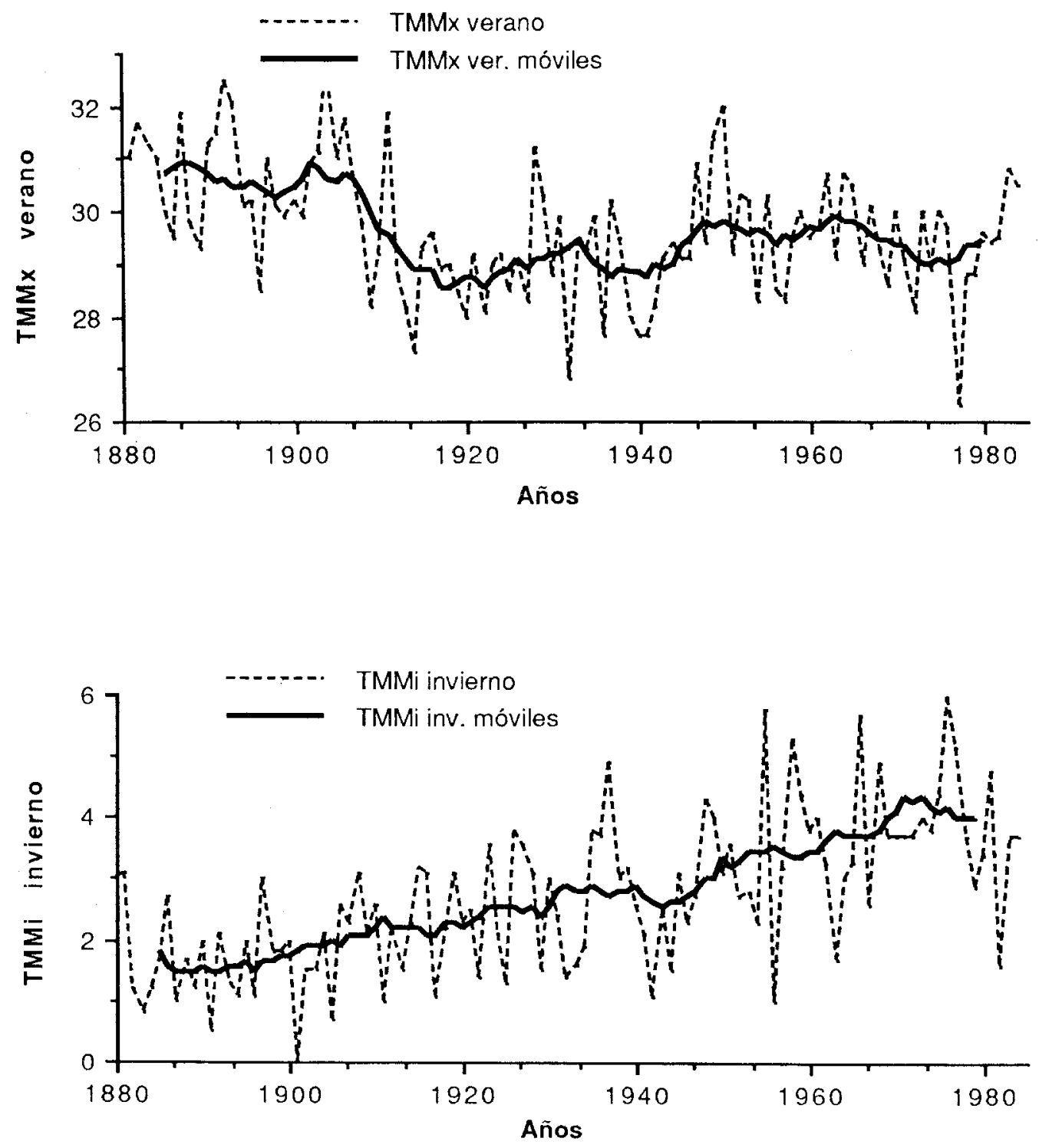

Fig. 3. Temperatura media de las máximas de verano (TMMx verano) y temperatura media de las mínimas de invicrno (TMMi invierno) de Zaragoza (1880-1984) y sus medias móviles de 11 años. 
En efecto, el enfriamiento estival parece ser la tónica dominante de los últimos cien años. El perfil térmico no es sencillo, muestra toda una serie de pequeñas alzas y bajas, pero progresivamente las temperaturas son más débiles. Asi lo contempla también la recta de tendencia: $\mathrm{t}_{\mathrm{i}}=30^{\prime} 27-0^{\prime} 013 \mathrm{xi}$, con un coeficiente de correlación hallado de - 0'54. De igual modo, si calculamos la temperatura media de las máximas registradas en dos períodos de alza, uno pasado y otro reciente, las diferencias son manifiestas:

Período 1891-1910: $30^{\prime} 5^{\circ}$ media de las máximas de verano.

Período 1951-1970: $25^{\circ}$ media de las máximas de verano.

A la inversa de lo que sucede en verano, el invierno es cada vez más cálido; pero además, con una tendencia mucho más perceptible y con una línea de continuo ascenso desde 1885 . El valor de las medias móviles ha pasado de $1^{\prime} 46^{\circ}$ que tenía en 1887 a 4'26 de 1973; es decir, se ha incrementado en $2^{\prime} 8^{\circ}$. La tendencia que dibuja el gráfico (ver figura 3 ) tiene, así mismo, un alto grado de significación, como prueba el valor del coeficiente de correlación: 0'92, calculado para obtener la ecuación de la recta de regresión, que equivale a: $t_{i}=1^{\prime} 394+00^{\prime} 027 x_{\mathbf{i}}$. También en este caso se pueden comparar registros de períodos diferentes y cronológicamente extremos, para apoyar más el comentario, y obtenemos estos resultados:

Período 1881-1900: $132^{\circ}$ media de las mínimas de invierno.

Período 1961-1980: 3'90 media de las mínimas de invierno.

Los efectos del aumento de temperatura se manifiestan de muy diverso modo. Así, el número anual de días de helada ha disminuido ostensiblemente, pasando de 31 días de promedio a finales del siglo XIX, a 16 días en la actualidad, lo que viene a suponer un $48 \%$ menos. Y, en cierto modo, lo mismo podríamos decir de los días de precipitación en forma de nieve, aunque este fenómeno está también relacionado con la disminución de la precipitación invernal.

La responsabilidad de esta variación térmica no puede atribuirse de manera exclusiva a la evolución climática general, sin duda la influencia del área urbana debe hacerse sentir en este cambio. La ciudad, como es bien sabido, ejerce un doble efecto sobre las temperaturas, positivo, sobre todo en invierno, por el aporte adicional de calor que generan las actividades urbanas y la propia morfología de la ciudad, y negativo frente a la radiación solar incidente. En Zaragoza, la acción sobre las temperaturas máximas no parece muy evidente, pero si en cambio en las temperaturas mínimas de invierno. Si comparamos la evolución de este observatorio con otros dos alejados del núcleo urbano situados en áreas rurales circundantes, como son el Aeropuerto de Zaragoza y el observatorio de Aula Dei, el aumento térmico desde 1960 ha sido difcrente y favorable a Zaragoza:

\author{
Zaragoza: $\quad 1^{\prime} 05^{\circ}$ de incremento \\ Aula Dei: $\quad 0^{\prime} 9^{\circ}$ \\ Aeropuerto: 0'80
}

Coincide este año, 1960, con el momento de mayor expansión urbana, con fuerte incremento de población y actividad económica, que ha propiciado la aparición de la "isla de calor" y la consiguiente modificación del clima. 


\section{CONCLUSION}

Este primer análisis de las temperaturas de Zaragoza ha permitido comprobar la existencia de varios ciclos bien definidos desde 1865, y sobre todo el ritmo térmico ascendente hasta 1950 y la posterior inversión de la tendencia. Tan sólo las temperaturas mínimas escapan a esta dinámica y permanecen estabilizadas en valores elevados. Lógicamente al descender las cifras máximas y mantenerso o subir las mínimas, se han suavizado las extremas y la amplitud anual sc ha reducido en más de $2^{\circ}$. El aumento más espectacular ha correspondido a las mínimas invernales, casi $3^{\circ}$ de incremento en un siglo, acentuado, además, a partir de 1943 como muestra la curva de las medias móviles. La explicación de estos hechos supone la existencia de un mecanismo de transporte de calor hacia el área templada, reforzado seguramente por la presencia de la "isla de calor urbana", y evidencia también que el examen de las causas posibles de estos cambios, actualmente en curso, es indispensable para la comprensión de estos fenómenos.

\section{BIBLIOGRAFIA CITADA}

CLAVERO, P. (1982): "Análisis climático de las temperaturas de Barcelona (Observatorio Fabra, período 19141981)": I Congrés d' Historia del Plá de Barcelona. pp. 55-63.

CREUS, J. y PUIGDEFABREGAS, J. (1981): "Fluctuaciones periodicas de una dendrocronología pirenaica". Estudia Aecologica, 1, pp. 131-140.

CREUS, J. y PUIGDEFABREGAS, J. (1983): "Climatología histórica y dendrocronología de Pinus Nigra". Av. Inv. Bioclimatologia, pp. 121-128.

ISSCOURROU, G. (1984): "L'evolution du climat français". Rev. Geo. de l'Est., no 1, pp. 17-26.

ESCOURROU, P. (1984): "Cent ans de climat nantais, 1881-1980". Rev. Geo. de l'Est., nº 1, pp. 79-88.

GONZALEZ HIDALGO, J.C. (1989): "Fluctuaciones climáticas de corto plazo: Necesidades de estudio en áreas de nuevos regadíos". Options Mediterranéennes, $\mathrm{n}^{\circ} 3$, pp. 331-335.

LAMB, H. (1982): Climate, History and the Modern World. Methuen, London, $387 \mathrm{p}$.

LANSBERG, H. (1984): "Global climatic trends", en SIMON, J. The resources ful Earth. A response to global 2000. Blacwell, pp. 272-315.

LOPEZ GOMEZ, A. y IFERNANDEZ, F. (1986): "Evolución térmica en Madrid durante el presente siglo". En LOPLZ,-VERA, Quaternary Climate in Western Mediterranean. pp. 249-269.

MORENO, M.C. y MARTIN VIDE, J. (1986): "Estudio preliminar sobre las tendencias de la precipitación anual en cl Sur de la Península Ibérica: el caso de Gibraltar". I Sym. Agua en Andalucia. pp. 37-44.

RASO, J.M. (1987): "Variaciones recientes de las temperaturas medias en el Observatorio del Ebro". Anales de Geografla de la Universidad Complutense, $\mathrm{n}^{\circ} 7$, pp. 155-165. 